\title{
Phase Transitions in Compounds of the Ln-M(II)-oda MOF Series
}

\author{
Guzman Andres Peinado ${ }^{1}$, Leopoldo Suescun ${ }^{2}$, Julia Torres $^{3}$, Carlos Kremer $^{3}$ \\ ${ }^{1}$ DETEMA, Facultad De Quimica (UdelaR), Montevideo, Uruguay, ${ }^{2}$ Cryssmat-Lab/DETEMA, Facultad de Quimica (UdelaR), \\ Montevideo, Uruguay, ${ }^{3}$ DEC, Facultad de Quimica (UdelaR), Montevideo, Uruguay \\ E-mail: gpeinado@fq.edu.uy
}

Metal-organic frameworks (MOFs) have been described as very promising materials for hydrogen (and other gases) storage due to their intrinsic open-framework characteristics and chemical flexibility related to the wide variety of possible materials. MOFs based in oxydiacetate (oda) as a ligand and containing lanthanide ions (Ln) and/or divalent metal ions (M) show multiplicity of structures dependent on the preparation route, the size of the lanthanide ion and the coordination properties of the metal. Two polymorphic series of these MOFs have been described, one with hexagonal symmetry [1-3] and another one with cubic symmetry [3]. The hexagonal form containing $\sim 1 \mathrm{~nm}$ size channels with potential gas storage capabilities shows an $M$ cation bonded to four oda and two water molecules, thus stabilized in the presence of very anisotropic cations such as $\mathrm{Cu}(\mathrm{II})$. The cubic form shows an atypical separation of $\mathrm{M}$ cations, two out of three forming part of the Ln-M-oda anionic network while the third one forming a hexa-acuo counter ion.

Phase transformations have been observed for some of the cubic compounds containing Co and Mn that are of crystallographic interest and may explain observed magnetic properties. Most of the compounds are radiation sensitive showing significant peak shape and intensity decay with absorbed dose. One initial diffraction experiment performed with a low intensity setup showed that Yb-Co phase converted from a rhombohedral phase at the beginning of the experiment to the expected cubic phase at the end. This rhombohedral phase evolved continuously towards the cubic phase with time converting completely into cubic after 8 hours of irradiation. The peaks from the rhombohedral phase merged into a single cubic peak in what appeared to be a continuous ( 2 nd order) phase transition. A second experiment aiming to compare the already irradiated sample with a freshly mounted one, but now using a high intensity diffractometer setup showed no signs of phase transformation but just the cubic phase for both samples.

Similar rhombohedral phase was observed for Gd-Mn compound, but in this case there is coexistence of rhombohedral and cubic phases at room temperature and the rhombohedral phase slowly converts to cubic on irradiation or heating. This transformation is somehow different from the one observed for $\mathrm{Yb}-\mathrm{Co}$ in the fact that phase coexistence is observed between initial and final states therefore the transformation may be 1st order. The two transformations observed close to room temperature for both $\mathrm{Yb}-\mathrm{Co}$ and $\mathrm{Gd}-\mathrm{Mn}$ may be related but are not identical.

Additional experiments on fresh $\mathrm{Yb}$-Co sample collected at $100 \mathrm{~K}$ show an additional phase transition from monoclinic to rhombohedral phase while collecting data. The clues for the stability of the different phases may reside on the detailed crystallographic structure. Precise determination of atomic positions of oxygen atoms (both from the ligand as from water molecules) was achieved to shed light on the factors controlling phase stability and also allow a better understanding of magnetic and gas storage properties of the different structural types.

[1] Kremer, C. et al. (2008) J. Mol. Struct. 879, 130

[2] Dominguez, S. et al. (2007) J. Mol. Struct. 829, 57.

[3] Chen, B. et al. (2007) Inorg. Chem. 46, 1233.

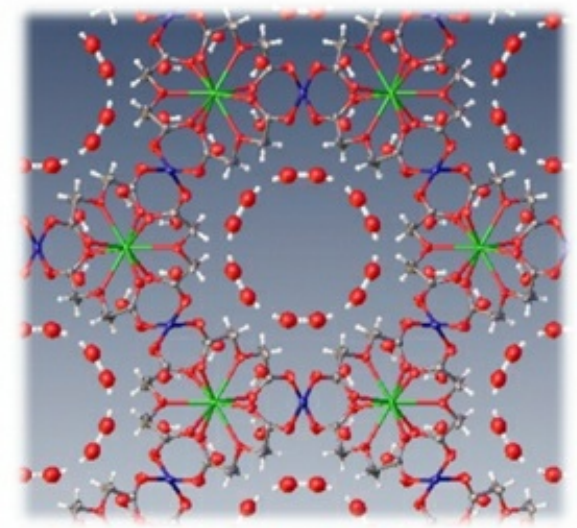

HEXAGONAL

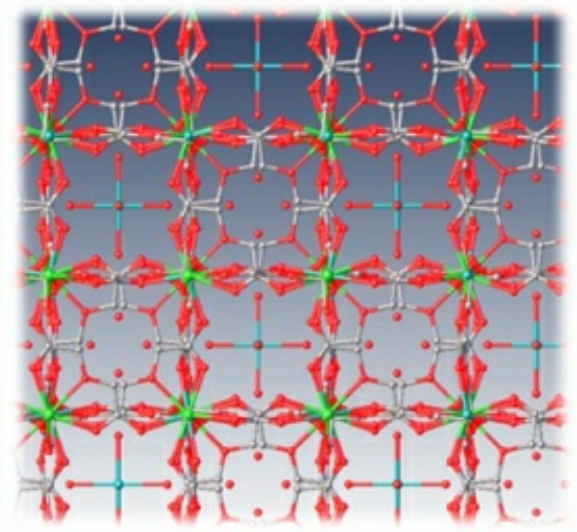

CUBIC

Keywords: lanthanide, MOF, phase transitions 